\title{
A!
}

This is an electronic reprint of the original article.

This reprint may differ from the original in pagination and typographic detail.

Tikkala, Vesa-Matti; Jämsä-Jounela, Sirkka-Liisa

\section{Monitoring of Caliper Sensor Fouling in a Board Machine Using Self-Organizing Maps}

\author{
Published in: \\ EXPERT SYSTEMS WITH APPLICATIONS
}

Published: 01/01/2012

Document Version

Peer reviewed version

Please cite the original version:

Tikkala, V-M., \& Jämsä-Jounela, S-L. (2012). Monitoring of Caliper Sensor Fouling in a Board Machine Using Self-Organizing Maps. EXPERT SYSTEMS WITH APPLICATIONS, 39(12), 11228-11233.

This material is protected by copyright and other intellectual property rights, and duplication or sale of all or part of any of the repository collections is not permitted, except that material may be duplicated by you for your research use or educational purposes in electronic or print form. You must obtain permission for any other use. Electronic or print copies may not be offered, whether for sale or otherwise to anyone who is not an authorised user. 


\title{
Monitoring of caliper sensor fouling in a board machine using self- organising maps
}

Vesa-Matti Tikkala, Sirkka-Liisa Jämsä-Jounela

Aalto University School of Chemical Technology, P.O. Box 16100, 00076 Aalto, Finland

\begin{abstract}
This paper presentes a process-monitoring scheme utilising adaptive self-organising maps (SOM) to detect process conditions that lead to the fouling of a caliper sensor in a board machine. The scheme is based on mapping on a SOM the process measurements and the calculated variables which provide insight into the chemical phenomena involved in fouling to classify faulty process conditions. The time-variant nature of the board making process was taken into account by regularly re-training the SOM. The monitoring scheme is demonstrated with industrial data, and the results are presented and discussed.
\end{abstract}

(C) 2012 Elsevier Ltd. All rights reserved.

Keywords

Process monitoring, Self-organising maps, Board machine, Quality control system, Caliper measurement, Fouling

\section{Introduction}

The increasing complexity of modern production processes, intensifying global competition, and environmental regulations are posing enormous challenges to the operation of production plants. They must run safely and efficiently, without any disturbances in operation or in the quality of their products.

Quality is particularly important in the pulp and paper industry, where high quality provides a significant competitive edge in the global markets. Quality control systems are therefore of great importance in modern paper- and board-making processes. Such systems have to be available for over $99 \%$ of the time in order to maintain uniform paper or board quality and to minimise production losses. Online measurements of the main quality variables are an essential part of quality control systems. As a consequence, the operation of these measurements at all times is a key factor in successful online control.

One of the most important quality variables in board making is the thickness of the board, its caliper. Caliper sensor types can be divided into two categories: magnetic and optical sensors. Magnetic sensors have two plates that contact the paper web on both sides and the distance between the plates is measured using magnetic reluctance (Tornberg, 1999). The main advantage of magnetic sensors is accuracy, but their contact with the web causes problems such as wear, web marking, and fouling. Optical caliper sensors, using either laser or light-based distance measurement, are a relatively new invention and several different constructions have been brought to the market in recent years (Williamson, 2011). Semi-contacting optical sensors, where the paper web is in contact with only one side of the web have been presented by ABB (Naimimohasses \& Hellstrom, 2009) and Metso (Graeffe \& Nuyan, 2005). ABB's sensor is based on a confocal displacement technique while Metso's sensor uses distance measurement based on laser triangulation. Completely non-contacting optical sensors which use confocal displacement and laser triangulation techniques have been developed by Voith (Voith Paper Automation, 2010) and Honeywell (Nakano, 2006), respectively. Optical caliper sensors have the obvious benefits of reduced web marking and fouling, but they are more expensive than magnetic sensors. Nevertheless, the magnetic sensor type 
remains the industrial standard in online caliper measurement (Williamson, 2011), and the related problems, such as fouling, are commonplace in the paper and board mills.

The fouling problem can be tackled without investing in new equipment by looking for solutions that make use of fault diagnosis and process monitoring. According to Isermann (2006), process monitoring is considered the continuous on-line task of determining the conditions of the process. This is typically achieved by means of process history data -based methods (see e.g. Venkatasubramanian, Rengaswamy, Kavuri, \& Yin, 2003), such as self-organising maps (SOM). SOMs were introduced by Kohonen (1982), and they have a wide variety of applications in different fields (Kangas \& Kaski, 1998) such as visualisation or voice and image analyses. They have also been successfully applied to process monitoring tasks such as the analysis of a wave soldering process (Liukkonen, Havia, Leinonen, \& Hiltunen, 2009), Internet-based remote supervision (Domínquez, Fuertes, Reguera, Díaz, \& Cuadrado, 2007), fault diagnosis of an ethylene cracking process (Kämpjärvi et al., 2008), and monitoring of a flash smelting furnace (Jämsä-Jounela, Vermasvuori, Endén, \& Haavisto, 2003), for instance.

In this paper, a SOM is utilised to monitor caliper sensor fouling in a board machine. The objectives of this case study are thus to study under which process conditions fouling occurs and to utilize a self-organising map to monitor the process and to estimate when fouling will occur.

This paper is organised as follows. Section 2 describes self-organising maps and their implementation. Section 3 describes the case process and the caliper sensor fault in question. The testing procedure and the results of the monitoring tests are presented in Sections 4 and 5, respectively followed by the conclusions in Section 6.

\section{Description of self-organising map}

A self-organising map is a type of artificial neural network that is trained using unsupervised learning to produce a low-dimensional representation of the input space of the training samples, called a map. A SOM produces a similarity graph of the input data by converting the nonlinear statistical relationships between high-dimensional data into simple geometric relationships on a low-dimensional display, usually a twodimensional grid of nodes. Therefore, SOM compresses the data, but preserves their topological properties (Kohonen, 1998).

A SOM consists of a number of neurons or nodes that are described with a d-dimensional weight vector (sometimes referred as a codebook vector)

$\mathbf{w}=\left[w_{1}, w_{2}, \ldots, w_{d}\right]$,

where $d$ denotes the dimension of the input data vectors (number of variables). The nodes are organised in the map according to a specific topology. The SOMs are typically presented as two-dimensional sheets in which the nodes are arranged in a rectangular or hexagonal lattice.

The self-organising map is trained by adapting the weights of the nodes to match the input data. The training can be either sequential training or batch training. Training basically consists of first determining the closest map units of the data samples, called the best-matching units (BMUs), and then updating the weight vector each BMU and its neighbouring nodes. The fundamental difference between the different training procedures is that the weights are updated after each sample in sequential training whereas the weights are updated once per epoch in batch training. The distance to the BMU $c$ for the data sample $\mathbf{x} \in \mathbb{R}^{d}$ is determined as follows: 
$\left\|\mathbf{x}-\mathbf{w}_{\mathbf{c}}\right\|=\min _{i}\left\{\left\|\mathbf{x}-\mathbf{w}_{\mathbf{i}}\right\|\right\}, \quad i=1, \ldots, m$,

where $\|\cdot\|$ is a distance measure, typically Euclidean, and $m$ is the number of map nodes.

After determining a BMU, the weight vector of the BMU and the neighbouring nodes are updated according to an update rule which typically has the following form:

$\mathbf{w}_{i}(t+1)=\mathbf{w}_{i}(t)+\alpha(t) h_{c i}(t)\left[\mathbf{x}(t)-\mathbf{w}_{i}(t)\right]$,

where $t$ denotes time, $h_{c i}(t)$ is the neighbourhood kernel around the BMU and $\alpha(t)$ is the learning rate. The neighbourhood kernel defines the effect of the update on the neighbouring nodes. The shape of the neighbourhood kernel and its radius determine the change of the weights of the nodes to be updated in each training step. Typically, the following Gaussian neighbourhood function is used:

$h_{c i}(t)=e^{-d_{c i}^{2} / 2 \sigma_{t}^{2}}$

where $d_{c i}=\left\|\mathbf{r}_{c}-\mathbf{r}_{i}\right\|$ is the distance between map nodes in the grid and $\sigma_{t}$ is the neighbourhood radius defining the width of the function.

In the batch training procedure, the BMUs are first calculated for the whole data set, and then the weights of the nodes are all updated at once. The data set is partitioned according to the Voronoi regions of the map units. That is, each input data vector $\mathbf{x}$ belongs to the data set of the map unit which it is closest to. Then the sum of the vectors in each Voronoi set is calculated as follows:

$\boldsymbol{s}_{i}(t)=\sum_{j=1}^{n_{V_{i}}} \mathbf{x}_{j}$

where $n_{V_{i}}$ is the number of samples in the Voronoi set of the node $i$. The new values of the weight vectors are calculated as according to

$\mathbf{w}_{i}(t+1)=\frac{\sum_{j=1}^{m} h_{i j}(t) \boldsymbol{s}_{j}(t)}{\sum_{j=1}^{m} n_{V_{j}}(t) h_{i j}(t)}$.

The SOM algorithm implementation (SOM Toolbox, Vesanto, Himberg, Alhoniemi, \& Parhankangas, 2000) used in this case study uses the batch training algorithm presented above. However, in order to reduce memory consumption, the best-matching units for each input data sample are not calculated all at once but in several batches. Nevertheless, the BMUs corresponding to each input data sample are determined before any adjustments to the weights are made.

The SOM is trained in two phases. In the rough training phase, which is performed first, a smaller number of training epochs and a larger neighbourhood radius are used than in the following fine-tuning phase.

\section{Description of the process and the caliper sensor fouling fault}

\subsection{Board making process}

The board making process begins with the preparation of raw materials in the stock preparation section. The different types of pulp are refined and blended according to a specific recipe in order to achieve the desired composition and properties for the board grade to be produced. The consistency of the stock is controlled by the addition of dilution water. 
The blended stock is pumped from the stock preparation to the short circulation by a pump that controls the basis weight of the board. In the short circulation, the stock is first diluted in the wire pit to the correct consistency for web formation. The diluted stock is then cleaned and screened before passing into the head box, from where it is sprayed onto the wire in order to form the paperboard web.

The excess water is first drained through the wire and further removed by pressing the board web between rolls in the press section. The remaining water is evaporated in the drying section using steam-heated drying cylinders. After the drying, the board is calendered in two phases in order to achieve the desired surface properties.

The important quality variables, such as basis weight, moisture, and caliper are measured after the calender section with a measurement scanner that traverses the board web.

\subsection{Caliper sensor fouling fault}

At the case board machine, caliper sensor fouling is a difficult problem, as reported by Jämsä-Jounela et al. (submitted for publication). In order to maintain the functionality of the quality control system, the sensor must be cleaned on a regular basis. Otherwise, fouling impedes the online control of the caliper profile and the monitoring of board caliper in the machine direction.

The caliper sensor is located in the measurement scanner. It consists of two plates that are in contact with each side of the web, see Fig. 1. The board travels between the plates and the distance between the plates can be measured by measuring the magnetic reluctance caused by the board caliper. Due to this construction, the sensor is subject to fouling. Dirt builds up on the sensor plates and disturbs the measurements.

Caliper measurements will drift significantly when fouling occurs. Fig. 2 shows the measurement data for $30 \mathrm{~h}$ in a faulty period. The figure clearly displays that the measured value increases with time, while the setpoint of the caliper was kept constant over the whole period. Between the cleanings of the sensor at hour 1 and at hour 27, the slope of the drift was approximately $1.2 \mathrm{~lm} / \mathrm{h}$.

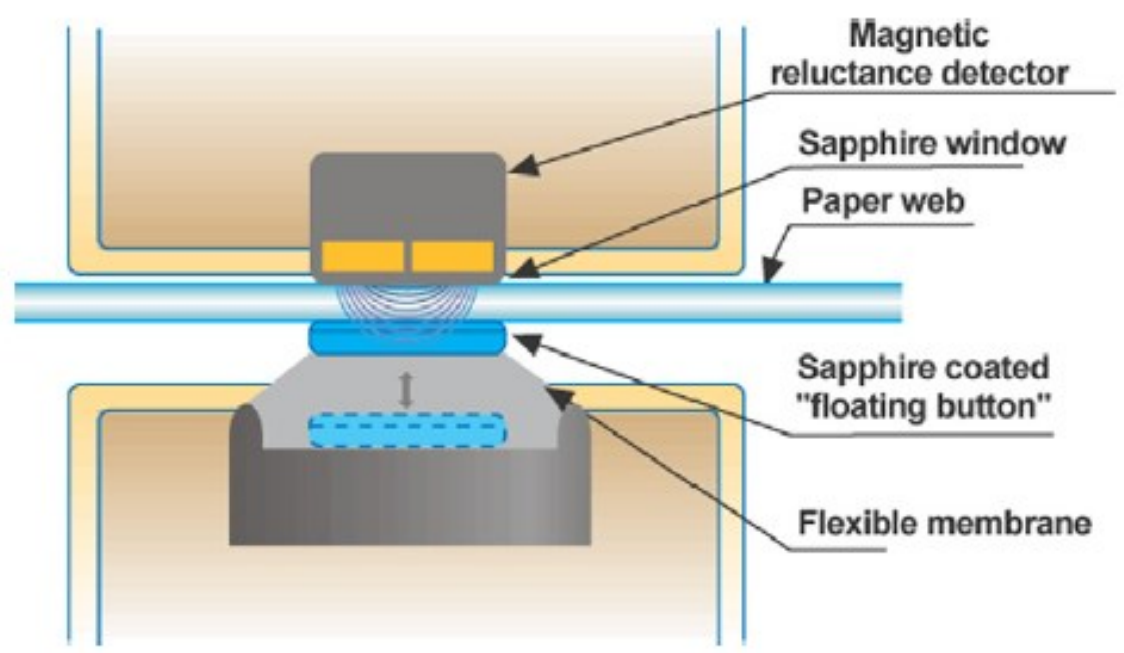

Fig. 1. Cross-sectional diagram of the caliper sensor. Modified from Anon (2009) 
According to maintenance experts, additional problems are caused by the flaking of the dirt. This typically takes place when the thickness of dirt layer reaches approximately $20 \mathrm{~lm}$. This then causes serious disturbances in the measurements.

To facilitate the mathematical analysis of the caliper sensor fault, a qualitative representation of the fault was needed. To this end, a binary variable called the fault indicator was developed based on the maintenance data of the board machine. According to the recorded maintenance actions on the caliper sensor, the fault indicator was given the values 1 or 0 to indicate faulty and normal operation, respectively. The measurement data of the caliper sensor was also examined to identify the periods with positive slopes, as shown in Fig. 2 .

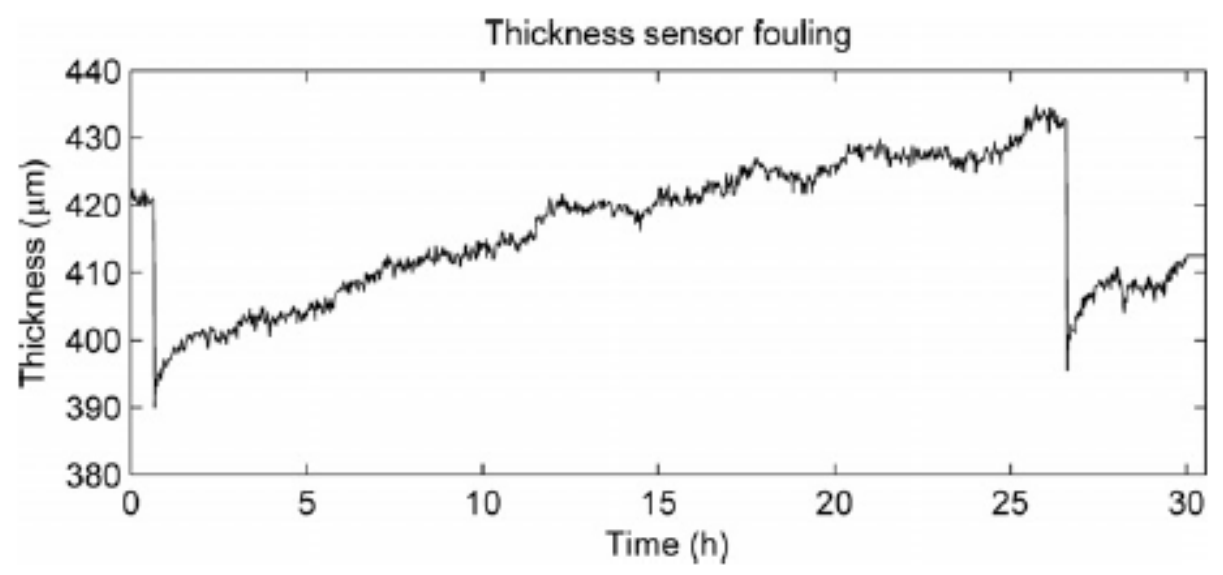

Fig. 2. Effect of the caliper sensor fouling. Data for $30 \mathrm{~h}$, showing the evolution of the caliper sensor readings between two cleanings (at hour 1 and at hour 27)

\section{Experiment description}

\subsection{Testing procedure}

The experiment was conducted as follows: First, the monitored variables were selected, and the calculated variables providing insight into the fouling phenomenon were developed. Next, a SOM was trained using a data set that contained faulty and normal operation data. Finally, the monitoring tests were carried out by introducing the test data sets to the SOM and calculating the best-matching units for each sample. The process conditions leading to caliper sensor fouling were detected by observing when faulty samples of the test data appeared on the map in similar locations to those of the training data.

\subsection{Selection of variables and development of calculated variables}

Variable selection is the most critical task when developing process monitoring systems. In this study, the variables were selected based on a correlation analysis, a SOM analysis, and process knowledge.

The correlation analysis aimed at exploring a large number of process variables to produce a set of candidates for further examination. The correlation analysis indicated that the web temperature (affected by the calender temperature, etc.) or the temperature surrounding the measurement scanner have an effect on fouling. In addition, certain chemicals used in the board production appeared to correlate with the fault.

Next, to confirm the results of the correlation analysis, the set of variable candidates was analysed using the SOM to study the interactions between the variables and their effect on the fouling phenomenon. 
It was discovered that neutral size is the main chemical affecting fouling (see also Tikkala, Myller, Kulomaa, Hämäläinen, \& Jämsä-Jounela, 2011).

Finally, to better take into account the chemical phenomena in fouling, the variable list was complemented with calculated variables related to the size chemical. The calculated variables are used to incorporate process knowledge into the monitoring methods (Komulainen, Sourander, \& Jämsä-Jounela, 2004). The first calculated variable $\mathrm{R}$ describes the chemical reaction between size molecules and wood fibres with an exponential function resembling the Arrhenius equation for the reaction rate constant. According to Neimo (1999), the reaction of size molecules with fibres is favoured by high $\mathrm{pH}$. Therefore, the exponential term is multiplied by the $\mathrm{pH}$ of the stock as follows:

$R=e^{-1 / T_{w}} \cdot \mathrm{pH}$,

where $T_{w}$ is the temperature of the web.

The second calculated variable, $C$, provides insight into curing, i.e. orientation phase, of the size molecules. Curing is favoured by high temperature and impeded by the moisture of the web (Neimo, 1999), leading to the following expression for $C$ :

$C=\frac{T_{W}}{M}$

where $M$ is the moisture of the web.

The last calculated variable $S$ describes the starch ratio of the stock. Since the amount of starch has a positive effect on the adsorption of the size particles (Neimo, 1999), $S$ was defined as

$S=\frac{F_{S}}{F_{t o p}}$

where $F_{S}$ is the starch flow and $F_{\text {top }}$ is the stock flow for the top layer.

The final list of variables for the SOM monitoring is presented in

Table 1.

Table 1

List of variables for the SOM analysis

\begin{tabular}{lll}
\hline$\#$ & Variable & Description \\
\hline 1 & $\mathrm{~F}$ & Caliper control error (cv-sp) \\
2 & $\mathrm{dF}$ & Filtered derivative of $\mathrm{F}$ \\
3 & $\mathrm{R}$ & Reaction of the size molecules and fibres \\
4 & $\mathrm{C}$ & Curing of the size molecules \\
5 & $\mathrm{~S}$ & Adsorption of size particles \\
6 & $\mathrm{~T}_{\text {cal }}$ & $1^{\text {st }}$ calender thermos roll temperature \\
7 & $\mathrm{P}_{0}$ & Zero-pressure level of the secondary hood \\
8 & $\mathrm{TH}_{\mathrm{H}}$ & Hood ventilation air temperature \\
9 & $\mathrm{FWS}_{\mathrm{WS}}$ & Wet strength size flow \\
10 & Fs & Starch flow \\
11 & $\mathrm{~F}_{\mathrm{NS}}$ & Neutral size flow \\
12 & FRS & Retention starch flow \\
13 & F & Retention agent flow \\
14 & TW & Temperature of the web
\end{tabular}




\subsection{Data preparation}

For training and testing purposes, six data sets were collected from the board machine and prepared. Each data set contained one month of data for the specific board grades with a sampling time of five minutes. The data sets consisted of 1200-3900 samples corresponding to 100-325 h of operation, as listed in Table 2.

Table 2

Data sets for the SOM monitoring tests

\begin{tabular}{llll}
\hline Data set & \# of samples & Hours & \% of faulty data \\
\hline$D_{1}$ & 1521 & 127 & 56.7 \\
$D_{2}$ & 2254 & 188 & 51.9 \\
$D_{3}$ & 1291 & 108 & 63.5 \\
$D_{4}$ & 2687 & 224 & 31.6 \\
$D_{5}$ & 2937 & 245 & 9.8 \\
$D_{6}$ & 3907 & 326 & 34.8 \\
\hline
\end{tabular}

The data was prepared by removing the non-production data, i.e. shut-downs and web breaks, and the outliers and by normalisation. The non-production data was removed by excluding data segments in which the production rate had a zero value. Outliers were removed manually by observing the data and by replacing the diverging values with the average of its neighbours. Finally, the data was normalised to reduce the effect of the measurement signals having different magnitudes. The prepared training data comprising the data sets D 1 and D2 is presented in Fig. 3.

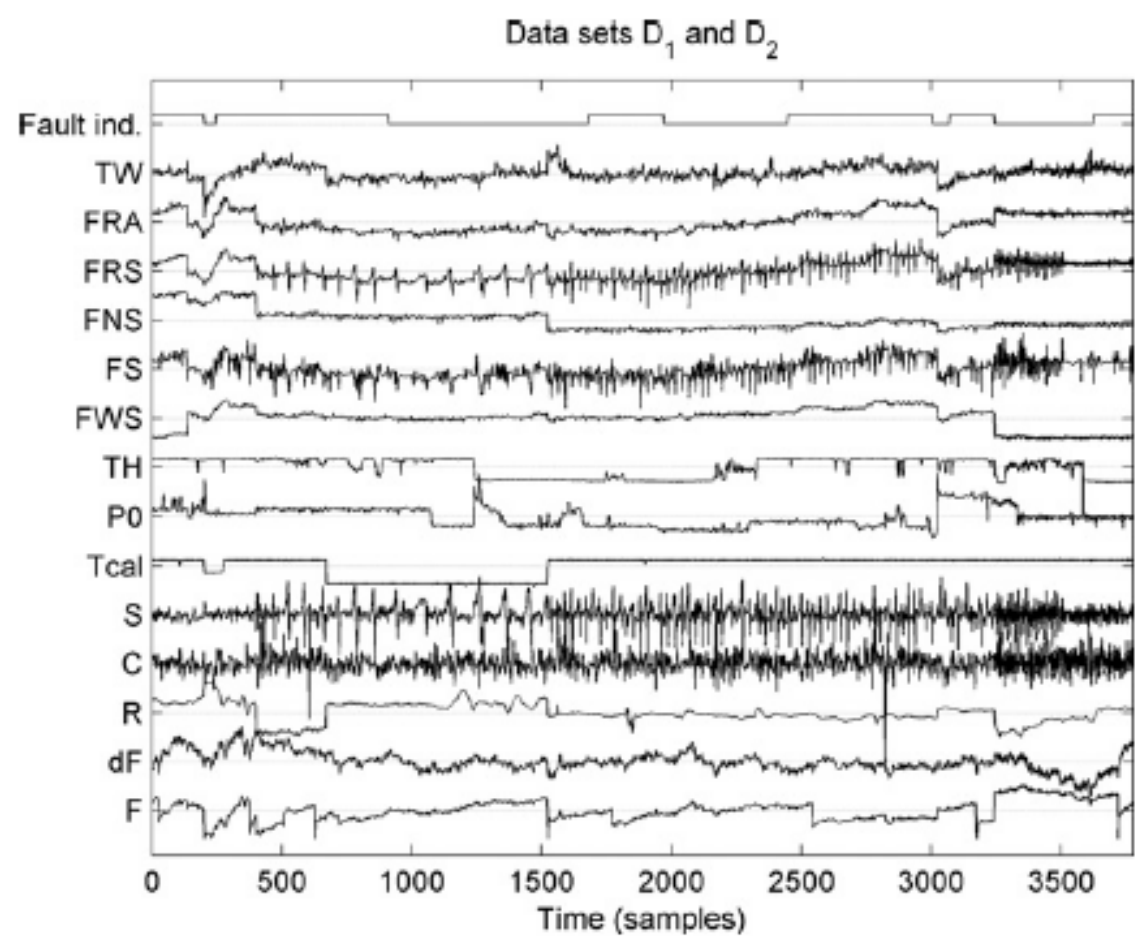

Fig. 3. Training data for the SOM. Data sets $D_{1}$ and $D_{2}$ 
The data samples were also labelled in order to separate the faulty operation from the normal operation. The labels for each sample were determined based on the fault indicator variable. Samples falling into faulty periods (fault indicator $=1$ ) were labelled ' $\mathrm{f}$ ' and the samples representing normal operation were labelled ' $n$ '. The labels were used to study the distribution of faulty and normal samples on the SOM.

\subsection{SOM training parameters}

The SOM for the monitoring tests was trained using an algorithm implemented for this purpose. The parameters used in the training SOM are listed in Table 3. The map size $\mathrm{m}$ was determined according to suggestions given in Vesanto et al. (2000). In addition, the number of training epochs and neighbourhood function radii were based on the default values in the literature (Vesanto et al., 2000) and were calculated according to the data and the size of the map. The map size was calculated as follows

$m=\operatorname{ceil}(5 \sqrt{n})$,

where $n$ is the number of samples in the training data. The map dimensions were then calculated using the eigenvalue decomposition of the training data matrix. The ratio of height $m_{h}$ to width $m_{w}$ of the map was determined as the ratio of the largest and the second largest eigenvalues. For the SOM used here, the map was found to consist of $m=77$ nodes organised in an 11-by-7 rectangular grid.

Table 3

Parameters of the SOM training

\begin{tabular}{lll}
\hline Parameter & Description & Value \\
\hline$m$ & Map size & 77 \\
$m_{h}, m_{w}$ & Map dimensions & {$[11,7]$} \\
& Initialization & Linear \\
& Training algorithm & Batch \\
& Number of training epochs, rough training & 1 \\
& Number of training epochs, finetuning & 1 \\
$h_{c i}$ & Neighbourhood kernel function & Gaussian \\
$\sigma_{t r}$ & Neighbourhood radius, rough training & 1.375 \\
$\sigma_{t f}$ & Neighbourhood radius, finetuning & 1 \\
\hline
\end{tabular}

The SOM was initialized by using a linear initialisation in which the initial map weight vectors lay in the subspace spanned by the two largest eigenvectors of the input data.

The lengths of the training phases, i.e. rough training and finetuning, were determined based on the size of the map and the amount of training data. A suggestion given in Vesanto et al. (2000) first defines the ratio of the number of map units $\mathrm{m}$ to the number of observation in the training data $n$ as follows:

$\xi=m / n$.

As a result, the used training length was $20 \cdot \xi$ of which $4 \cdot \xi$ was for rough training and $16 \cdot \xi$ for finetuning.

The neighbourhood function used by the algorithm is a Gaussian kernel function, see (4). Its radius was separately defined for each training phase. Typically, a larger neighbourhood radius is used in the rough training than in the finetuning phase. For the rough training phase, the neighbourhood radius was determined as follows: 
$\sigma_{t r}=\max \left(1, \max \left(\left[m_{h} m_{w}\right]\right) / 8\right)$.

According to (12), the rough training neighbourhood radius was determined to be $\sigma_{t r}=1.375$, and $\sigma_{t f}=$ 1 was used as the fine-tuning phase radius.

In order for the SOM to adapt to the varying conditions of the board machine process, the SOM was retrained after testing with each data set. The re-training phase consisted of one finetuning step, where the weights of the previous map were given as initial values for the new one and a short neighbourhood radius was used, $\sigma_{t f}=1$.

\section{Monitoring results}

The monitoring tests were carried out as follows. First, a SOM was trained using $\mathrm{D}_{1}$ and $\mathrm{D}_{2}$, and the set of faulty map units $\mathrm{F}$, indicating the faulty regions of the map, was identified. Next, $\mathrm{D}_{3}$ was introduced, and the BMUs for each sample were computed. The samples, whose BMUs belonged to F were associated with the faulty process conditions. Finally, the map was re-trained using $\mathrm{D}_{2}$ and $\mathrm{D}_{3}$, and the tests were carried out using $\mathrm{D}_{3}$. The rest of the data was treated similarly.

Fig. 4 illustrates the results of the SOM training using the data sets $\mathrm{D}_{1}$ and $\mathrm{D}_{2}$. The top left panel of the figure, the unified distance matrix (U-matrix), displays the clustering of the training data, where dark colours indicate short distances between the data samples. The single variable maps describe the distribution of high and low values of each variable on the map. Finally, the bottom right panel shows the distribution of faulty and normal data on the map. A major cluster of faulty samples is located in the middle of the left hand side of the map. In addition, a minor faulty cluster is located in the bottom left corner of the map. In conclusion, the effects of the temperature variables and the chemicals are clearly visible in Fig. 4.

The results of the monitoring tests using the SOM are presented in Fig. 5, where the panels from top to bottom show the results for the data sets $\mathrm{D}_{3}, \mathrm{D}_{4}, \mathrm{D}_{5}$, and $\mathrm{D}_{6}$, respectively. To reduce noise and false alarms, the estimated state has been filtered using a moving average filter with a window length of 5 samples. By means of the comparison presented in Fig. 5, it can be confirmed that the SOM gives a rather good estimate of the actual process conditions. Caliper sensor fouling is detected in most of the cases. Especially when fouling has been occurring for a longer time period, as demonstrated with the data sets $\mathrm{D}_{3}$ and $\mathrm{D}_{6}$, the $\mathrm{SOM}$ is able to detect the conditions correctly. Difficulties with the detection however arise in shorter faulty periods.

The performance of the SOM is summarised by computing the rates of correctly estimated states, falsely estimated states and uncertain states; see Table 4. Based on the monitoring tests, the SOM is on average able to estimate the state of the process correctly in over $70 \%$ of the time. The rate of falsely estimated states is rather low, on average below $20 \%$.

The perceived errors may result from the fault indicator, which has been created based on the dates of the maintenance reports and then confirmed by visual inspection of the data. As a result, the fault indicator might not be exactly aligned with actual fouling.

Table 4

Results of the monitoring tests using the SOM

\begin{tabular}{lllll}
\hline & $\mathrm{D}_{3}(\%)$ & $\mathrm{D}_{4}(\%)$ & $\mathrm{D}_{5}(\%)$ & $\mathrm{D}_{6}(\%)$ \\
\hline Correct process states & 61.7 & 67.0 & 86.2 & 19.8 \\
False process states & 19.0 & 29.0 & 10.8 & 19.8 \\
Uncertain process states & 19.3 & 4.0 & 3.0 & 10.3 \\
\hline
\end{tabular}




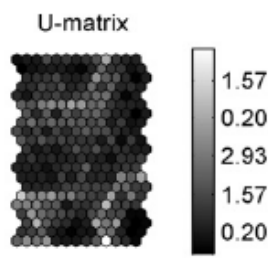

C

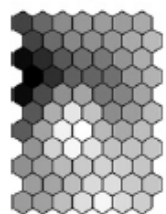

TH

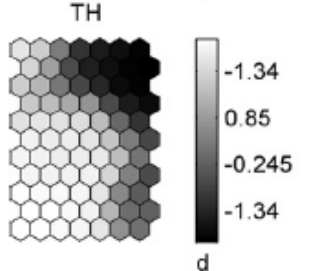

FRS
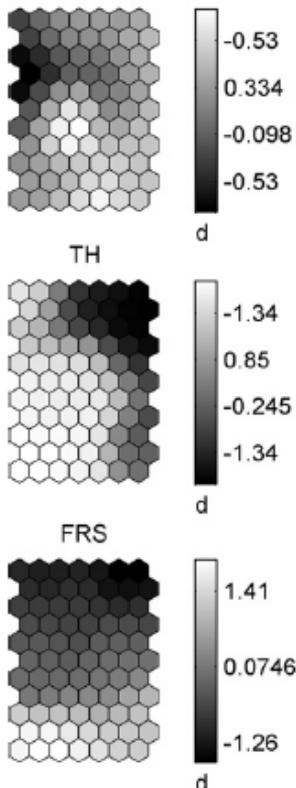

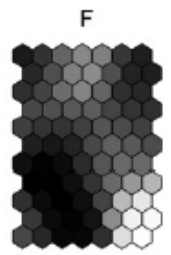

S

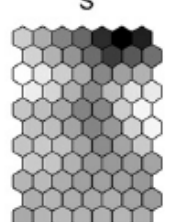

FWS

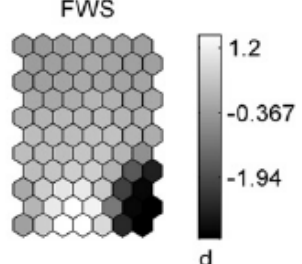

FRA

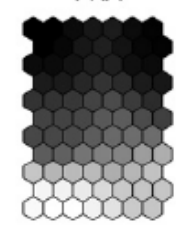

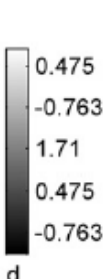
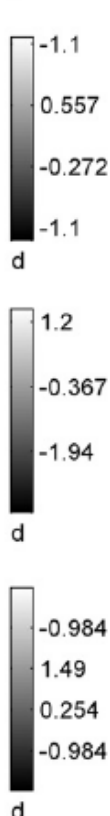

$\mathrm{dF}$

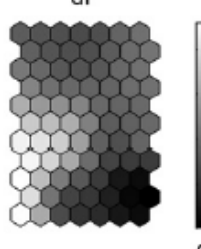

Tcal

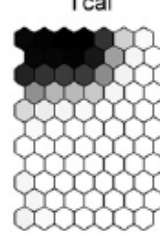

FS

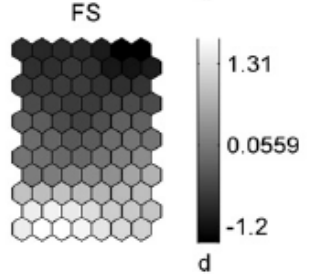

TW

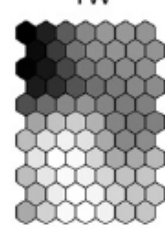

R

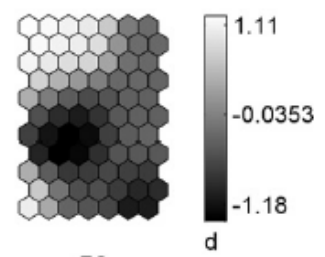

PO

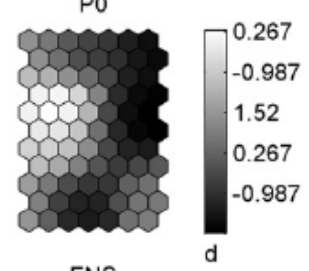

FNS

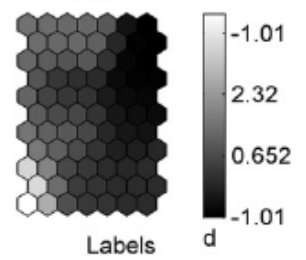

innm nnininn

Inininin In

nininin in in

in In f in in

nif Inininin

Ofininin

f f f f in non

if in in in

in ininin

fininin in in

Fig. 4. Overview of the SOM analysis for the training data: U-matrix, single variables maps and distribution of the faulty and normal operation samples
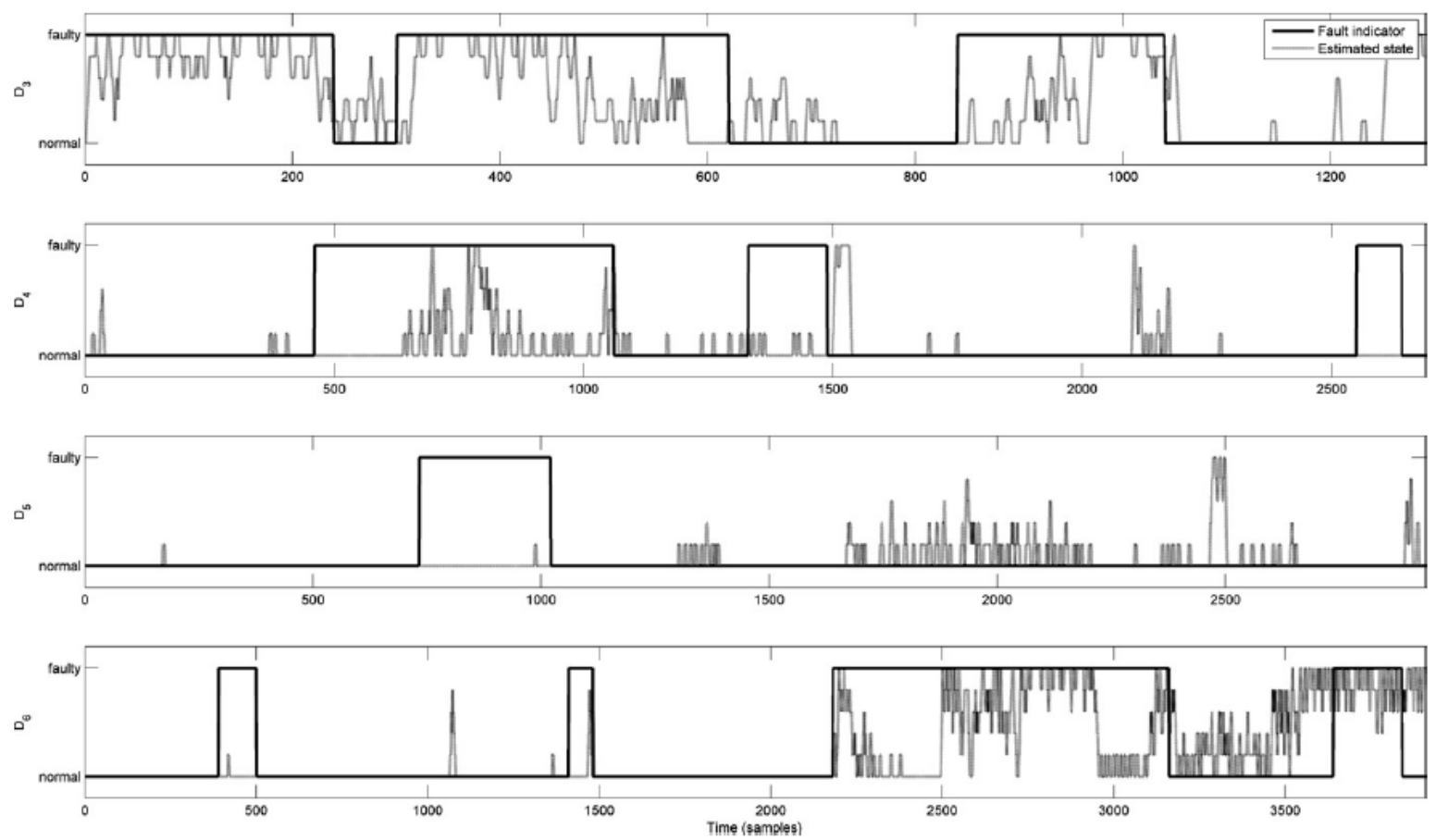

Fig. 5. The monitoring results using the SOM. From top to bottom: $D_{3}, D_{4}$, and $D_{6}$ 


\section{Conclusion}

In this study, a SOM-based monitoring scheme was developed for caliper sensor fouling on a board machine. Process measurements and calculated variables which describe the chemical phenomena involved in fouling were monitored and the state of the process was determined based on the classifications provided by the self-organising map. The scheme was tested with industrial data collected from the plant and its performance was evaluated.

The obtained results are promising. They show that the SOM is able to detect the conditions leading to fouling of the caliper sensor. This means that an indicator can be developed which informs the operators of the process of conditions in which it is likely to develop problems in caliper measurements. The operators can then take counteractions by scheduling maintenance or by changing the operating point if possible.

In a future work, the results can be improved by acquiring data that is more accurate on the fouling phenomenon itself. Information such as the rate of fouling in different operating conditions can be obtained by means of regular inspections of the caliper sensor to measure the dirt layer adhering to the sensor surfaces, for instance. Such knowledge would enable a more accurate classification of process conditions. In addition, the calculated variables can be improved through a more rigorous study of the chemical reactions involved in the interactions of neutral size and board fibres.

\section{Acknowledgements}

The research leading to these results has received funding from the European Union Seventh Framework Programme (FP7/2007-2013) under Grant Agreement No. 257580. The authors wish to acknowledge the support of the research consortium. In particular, the authors wish to thank Stora Enso Oyj and Efora Oy for providing the data and the expert knowledge for the analyses.

\section{References}

Anon (2009). Knowpap 11.0 - Learning environment for papermaking and automation.

Domínquez, M., Fuertes, J., Reguera, P., Díaz, I., \& Cuadrado, A. (2007). Internet-based remote supervision of industrial processes using self-organizing maps. Engineering Applications of Artificial Intelligence, 20, 757-765.

Graeffe, J., \& Nuyan, S. (2005). An online laser caliper measurement for the paper industry. In Optical measurement systems for industrial inspection IV. Proceedings of SPIE (Vol. 5856).

Isermann, R. (2006). Fault-diagnosis systems: An introduction from fault detection to fault tolerance. Springer.

Jämsä-Jounela, S.-L., Tikkala, V.-M., Laavi, H., Myller, T., Kulomaa, T., \& Hämäläinen, V. (submitted for publication). Outline of a fault diagnosis system for a largescale board machine. International Journal of Advanced Manufacturing Technology.

Jämsä-Jounela, S.-L., Vermasvuori, M., Endén, P., \& Haavisto, S. (2003). A process monitoring system based on the Kohonen self-organizing maps. Control Engineering Practice, 11, 83-92.

Kämpjärvi, P., Sourander, M., Komulainen, T., Vatanski, N., Nikus, M., \& Jämsä-Jounela, S.-L. (2008). Fault detection and isolation of an on-line analyzer for an ethylene cracking process. Control Engineering Practice, 16, 1-13.

Kangas, J., \& Kaski, S. (1998). 3043 Works that have been based on the self-organizing map (SOM) method developed by Kohonen. Technical report A49.

Kohonen, T. (1982). Self-organized formation of topologically correct feature maps. Biological Cybernetics, 43, 59-69.

Kohonen, T. (1998). The self-organizing map. Neurocomputing, 21, 1-6. 
Komulainen, T., Sourander, M., \& Jämsä-Jounela, S.-L. (2004). An online application of dynamic PLS to a dearomatization process. Computers \& Chemical Engineering, 28, 2611-2619.

Liukkonen, M., Havia, E., Leinonen, H., \& Hiltunen, Y. (2009). Application of selforganizing maps in analysis of wave soldering process. Expert Systems with Applications, 36, 4604-4609.

Naimimohasses, R., \& Hellstrom, A. (2009). Next generation optical caliper sensor technology. In TAPPI Papercon'09 conference, St. Louis, USA, May 31-June 1.

Nakano, R. (2006). Non contacting caliper measurements (laser caliper sensor). Japan TAPPI Journal, 60, $169-175$.

Neimo, L. (1999). Internal sizing of paper. In L. Neimo (Ed.), Papermaking chemistry (pp. 151-203). FAPET Oy.

Tikkala, V.-M., Myller, T., Kulomaa, T., Hämäläinen, V., \& Jämsä-Jounela, S.-L. (2011). Data analysis and monitoring of thickness sensor fouling using self-organized maps. In 1st Papyrus workshop on fault diagnosis and fault-tolerant control, Porticcio, France, October 6-7.

Tornberg, J. (1999). Special measurements in pulp and paper processes. In K. Leiviskä (Ed.), Process control (pp. 49-71). FAPET Oy.

Venkatasubramanian, V., Rengaswamy, R., Kavuri, S. N., \& Yin, K. (2003). A review of process fault detection and diagnosis, Part III: Process history based methods. Computers and Chemical Engineering, 27, 327-346.

Vesanto, J., Himberg, J., Alhoniemi, E., \& Parhankangas, J. (2000). SOM Toolbox for Matlab 5. Technical report A57.

Voith Paper Automation (2010). At last: A revolutionary non-contacting caliper measurement, Carta \& Cartiere (pp. 50-52).

Williamson, M. (2011). Optical caliper sensing comes of age. Pulp \& Paper International. 